\title{
AN OPPORTUNISTIC STBC-OFDM SCHEME WITH REDUCED PAR IN THE PRESENCE OF FREQUENCY OFFSET
}

\author{
Dandan Wang, Hlaing Minn and Naofal Al-Dhahir \\ Dept of Electrical Engineering \\ University of Texas at Dallas, Richardson, Tx 75083 \\ Email: dxw053000, hlaing.minn, aldhahir@utdallas.edu
}

\begin{abstract}
In [1][2][3], opportunistic space-time block codes were presented for flat-fading channels. In this paper, we propose an opportunistic STBC-OFDM for frequency-selective channels with an unequal error protection capability making it attractive for multimedia applications. Our new scheme optimizes the tradeoff between coding gain and peak-to-average ratio while minimizing inter-carrier interference in the presence of carrier frequency offset. We show that our scheme achieves a $2 \mathrm{~dB}$ reduction in PAR over the conventional scheme.
\end{abstract}

Index Terms- STBC, OFDM, PAR, ICI

\section{INTRODUCTION}

Space-time block coding (STBC) has received considerable attention in academic and industrial circles due to its many advantages [4][5][6]. Recently, diversity-embedding space-time codes (DEC) were proposed to embed a highdiversity code within a high-rate code [1][2][3]. They encode the information symbols into layers each at a prescribed rate-diversity operating point, thus providing an unequal error protection capability. This allows a form of wireless communications where the high-rate code opportunistically takes advantage of good channel realizations while the embedded high-diversity code ensures that at least part of the information is decoded reliably. However, previous DEC constructions were presented for flat-fading channels only. To utilize the frequency diversity gain of the frequencyselective channels, STBCs have been combined with orthogonal frequency division multiplexing (OFDM) by implementing STBC at a block level instead of symbol level, namely STBC-OFDM [7]. A straightforward approach to combine DEC with OFDM is to use the same structure of STBC-OFDM. However, this will cause high peak to average ratio (PAR), requiring a larger backoff and hence power loss at nonlinear amplifiers. Our proposed opportunistic STBCOFDM is developed by minimizing the PAR and the effects of carrier frequency offset by means of switching the signals on the time slots of DEC for some sub-carriers.

\section{PAR REDUCTION DESIGN FOR OPPORTUNISTIC STBC-OFDM}

In this section, we describe our proposed opportunistic STBC-OFDM scheme. We first focus on the following DEC design for 2 transmit antennas [3]

$$
G=\left(\begin{array}{cc}
a(0) & \frac{b(0)}{K} \\
-\frac{b^{*}(1)}{K} & -a^{*}(0)
\end{array}\right),
$$

where $(.)^{*}$ denotes complex conjugation. Information symbols are transmitted in 2 layers. Layer A consists of $a(0)$ while layer $\mathrm{B}$ consists of $b(0)$ and $b(1)$ resulting in an overall rate of 1.5 symbols per channel use. The scalar $K$ is designed to ensure a diversity level of 2 for layer A and 1 for layer B. In this paper, we choose all the signals from a unit-energy QPSK constellation. Extensions to other constellations are straightforward. It is interesting to note that if we switch the two signals on the two time slots of DEC, i.e.

$$
G^{\prime}=\left(\begin{array}{cc}
\frac{b(0)}{K} & a(0) \\
-a^{*}(0) & -\frac{b^{*}(1)}{K}
\end{array}\right),
$$

the switched DEC (S-DEC) maintains the same performance as DEC since it can be easily switched back at the receiver. Motivated by this observation, we propose our new opportunistic STBC-OFDM scheme which is illustrated in Fig. 1. The basic idea of our scheme is to implement hybrid DEC/SDEC at the OFDM symbol level. Let $J_{a}$ denote the set of the sub-carriers on which S-DEC is implemented while $J_{b}$ denote the set of sub-carriers on which DEC is transmitted. Suppose the size of $J_{a}$ is $m$ and let $N$ denote the size of an OFDM symbol. In addition, let $a_{0, n}, b_{0, n}$ and $b_{1, n}$ denote the signals $a(0), b(0)$ and $b(1)$, respectively, associated with the $n$-th $(n=0,1, \ldots, N-1)$ sub-carrier.

Consider the first transmit antenna. From (1) and (2), we obtain the signal transmitted on the $n$th subcarrier in the first time slot as

$$
s_{1, n}=\left\{\begin{array}{c}
\frac{b_{0, n}}{K}, n \in J_{b} \\
a_{0, n}, n \in J_{a}
\end{array}\right.
$$

and in the second time slot as

$$
s_{2, n}=\left\{\begin{array}{l}
a_{0, n}, n \in J_{b} \\
\frac{b_{0, n}}{K}, n \in J_{a}
\end{array} .\right.
$$




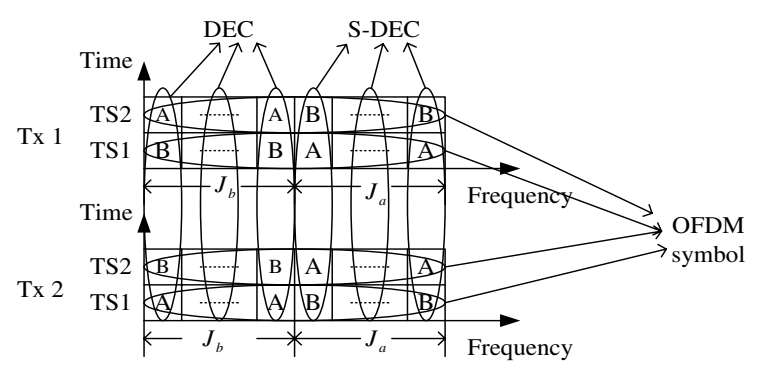

Fig. 1. Illustration of one STBC-OFDM block

Let $\left\{\hat{s}_{1, p}\right\}$ and $\left\{\hat{s}_{2, p}\right\}$ denote the low-pass-equivalent discrete-time signals obtained with an over-sampling factor of $M$ from the first and second OFDM symbols, respectively. Then,

$$
\begin{aligned}
\hat{s}_{1, p} & =\frac{1}{M N}\left\{\sum_{n=0, n \in J_{b}}^{N-1} \frac{b_{0, n}}{K} e^{\frac{j 2 \pi n p}{M N}}+\sum_{n=0, n \in J_{a}}^{N-1} a_{0, n} e^{\frac{j 2 \pi n p}{M N}}\right\}, \\
p & =0,1, \ldots, M N-1,
\end{aligned}
$$

whose amplitudes are upper bounded as

$$
\begin{aligned}
\left|\hat{s}_{1, p}\right| & \leq \frac{1}{M N}\left\{\sum_{n=0, n \in J_{b}}^{N-1}\left|\frac{b_{0, n}}{K}\right|\left|e^{\frac{j 2 \pi n p}{M N}}\right|+\sum_{n=0, n \in J_{a}}^{N-1} \mid a_{0, n} \| e^{\frac{j 2 \pi n p}{M N} \mid}\right\} \\
& =\frac{1}{M N}\left(\frac{N-m}{K}+m\right),
\end{aligned}
$$

and

$$
\left|\hat{s}_{2, p}\right| \leq \frac{1}{M N}\left(\frac{m}{K}+N-m\right) .
$$

Let $\mathbf{c}=\left\{\hat{s}_{1,0}, \hat{s}_{1,1}, \ldots, \hat{s}_{1, M N-1}, \hat{s}_{2,0}, \hat{s}_{2,1}, \ldots, \hat{s}_{2, M N-1}\right\}$ denote the signals transmitted on the first antenna during one STBC-OFDM block and $c(p)$ represent the $p$ th element of c. By using a sufficiently large $M$, the PAR of the continuoustime STBC-OFDM symbol can be approximated as

$$
\mathrm{PAR}=\frac{\max _{p}|c(p)|^{2}}{\frac{1}{2 M N} \sum_{p=0}^{2 M N-1}|c(p)|^{2}} .
$$

According to the Parseval's theorem, we have $\frac{\sum_{n=0}^{N-1}\left|s_{1, n}\right|^{2}}{M N}=$ $\sum_{p=0}^{M N-1}\left|\hat{s}_{1, p}\right|^{2}$ and $\frac{\sum_{n=0}^{N-1}\left|s_{2, n}\right|^{2}}{M N}=\sum_{p=0}^{M N-1}\left|\hat{s}_{2, p}\right|^{2}$. Then, we obtain

$$
\frac{1}{2 M N} \sum_{p=0}^{2 M N-1}|c(p)|^{2}=\frac{\frac{\frac{N-m}{K^{2}}+m+\frac{m}{K^{2}}+N-m}{N M}}{2 N M}=\frac{1+\frac{1}{K^{2}}}{2 N M^{2}} .
$$

Thus, the maximum PAR over all possible codewords is

$$
\begin{aligned}
\mathrm{PAR}_{\max } & =\max _{a_{0, n}, b_{0, n}, b_{1, n}}\{\mathrm{PAR}\} \\
& = \begin{cases}\frac{2\left(\frac{m}{K}+N-m\right)^{2}}{N\left(1+\frac{1}{K^{2}}\right)} & m \leq \frac{N}{2} \\
\frac{2\left(\frac{N-m}{K}+m\right)^{2}}{N\left(1+\frac{1}{K^{2}}\right)} & m \geq \frac{N}{2}\end{cases}
\end{aligned}
$$

The optimum $m$ is obtained by minimizing the maximum PAR as follows

$$
m_{\mathrm{opt}}=\underset{m}{\operatorname{argmin}}\left\{\mathrm{PAR}_{\max }\right\}=\frac{N}{2},
$$

and the corresponding maximum PAR is

$$
\mathrm{PAR}_{\max }=\frac{N(K+1)^{2}}{2\left(1+K^{2}\right)} .
$$

Similarly, we obtain the same PAR on the second transmit antenna as in (12). Therefore, to obtain the minimum PAR, DEC and S-DEC should be each transmitted on half of the sub-carriers (we call this opportunistic STBC-OFDM scheme as S-DEC-OFDM henceforth). Note that when $m=$ 0 , DEC will be transmitted on all the sub-carriers (we call it DEC-OFDM), which has the same structure as STBCOFDM. From (10) assuming $K>1$ as defined in [3], we can obtain that $\mathrm{PAR}_{\max }$ for DEC-OFDM is $\frac{2 N K^{2}}{1+K^{2}}$, which is much larger than the PAR of our proposed scheme in (12). Using the same approach, we can design opportunistic STBC-OFDM schemes for 3 and 4 transmit antennas. Due to space limitation, we will not discuss them in details in this paper.

\section{ICI REDUCTION}

In the previous section, we proposed an opportunistic STBC-OFDM scheme and proved that the lowest PAR is obtained by transmitting each of DEC/S-DEC on half of the sub-carriers. In this section, we will specify on which sub-carriers the DEC and S-DEC should be implemented by investigating the inter-carrier interference (ICI) introduced by the carrier frequency offset.

Let $\left\{h_{l}: l=0, . ., L-1\right\}$ and $\left\{g_{l}: l=0, . ., L-1\right\}$ denote two independent circularly-symmetric channels corresponding to the first and the second transmit antennas, respectively. The channel tap gains are assumed to be uncorrelated and constant over two adjacent OFDM symbols. The signal transmitted on the $n$-th sub-carrier in the first time slot on the first antenna is $s_{1, n}$ as given in (3). Then, the signal transmitted on the $n$-th sub-carrier in the first time slot on the second antenna is $\bar{s}_{1, n}$ given by

$$
\bar{s}_{1, n}=\left\{\begin{array}{l}
-a_{0, n}^{*}, n \in J_{b} \\
-\frac{b_{1, n}^{*}}{K}, n \in J_{a} .
\end{array}\right.
$$

Thus, the low-pass-equivalent received sample at the first time slot after the cyclic prefix removal is

$$
\begin{gathered}
y_{i}=\frac{1}{N} e^{\frac{j 2 \pi i v}{N}} \sum_{l=0}^{L-1} h_{l} \sum_{n=0}^{N-1} s_{1, n} e^{\frac{j 2 \pi(i-l) n}{N}}+ \\
\frac{1}{N} e^{\frac{j 2 \pi i v}{N}} \sum_{l=0}^{L-1} g_{l} \sum_{n=0}^{N-1} \bar{s}_{1, n} e^{\frac{j 2 \pi(i-l) n}{N}}+w_{i},
\end{gathered}
$$

where $v$ is a frequency offset (normalized by the subcarrier spacing) introduced by oscillators' inaccuracies and 
Doppler shift of the mobile wireless channel, and $\left\{w_{i}\right\}$ are independent, circularly-symmetric, zero-mean complex Gaussian noise samples. After FFT operation, the received $n$-th sub-carrier symbol is given by

$$
\begin{aligned}
& Y_{n}=I_{0} s_{1, n} H_{n}+\sum_{j=0, j \neq n}^{N-1} I_{j-n} s_{1, j} H_{j}+ \\
& I_{0} \bar{s}_{1, n} G_{n}+\sum_{j=0, j \neq n}^{N-1} I_{j-n} \bar{s}_{1, j} G_{j}+W_{n} \\
& \quad \equiv I_{0} s_{1, n} H_{n}+\mathcal{I}_{1, n}+I_{0} \bar{s}_{1, n} G_{n}+\mathcal{I}_{2, n}+W_{n},
\end{aligned}
$$

where $H_{n}$ and $G_{n}$ are the $n$-th sub-carrier channel gains from the first and second transmit antennas, respectively, $\left\{W_{n}\right\}$ are frequency-domain Gaussian noise samples corresponding to the time-domain noise samples $\left\{w_{i}\right\}$, and $I_{j-n}$ is given by

$$
I_{j-n}=\frac{1}{N} \sum_{m-1}^{N-1} e^{\frac{j 2 \pi(v+j-n) m}{N}} .
$$

As all the signals are uniformly chosen from QPSK constellation, $E\left\{s_{1, m} \bar{s}_{1, n}\right\}=0$ and the ICI becomes

$$
\begin{aligned}
& E\left\{\mathcal{I}_{1, n}+\mathcal{I}_{2, n}\right\}^{2}=E\left\{\left(\mathcal{I}_{1, n}\right)^{2}\right\}+E\left\{\left(\mathcal{I}_{2, n}\right)^{2}\right\} \\
& =E\left\{\sum_{j=0, j \neq n}^{N-1} \sum_{m=0, m \neq n}^{N-1} I_{j-n} s_{1, j} H_{j} I_{m-n}^{*} s_{1, m}^{*} H_{m}^{*}\right\}+ \\
& E\left\{\sum_{j=0, j \neq n}^{N-1} \sum_{m=0, m \neq k}^{N-1} I_{j-n} \bar{s}_{1, j} G_{j} I_{m-n}^{*} \bar{s}_{1, m}^{*} G_{m}^{*}\right\} .
\end{aligned}
$$

Since

$$
E\left\{s_{1, m} s_{1, n}^{*}\right\}=\left\{\begin{array}{cc}
1 & m=n \in J_{a} \\
\frac{1}{K^{2}} & m=n \in J_{b} \\
0 & m \neq n .
\end{array}\right.
$$

and

$$
E\left\{\bar{s}_{1, m} \bar{s}_{1, n}^{*}\right\}=\left\{\begin{array}{cc}
\frac{1}{K^{2}} & m=n \in J_{a} \\
1 & m=n \in J_{b} \\
0 & m \neq n .
\end{array}\right.
$$

the ICI variance in (17) can be expressed as follows

$$
\begin{aligned}
& E\left\{\left(\mathcal{I}_{1, n}\right)^{2}\right\}+E\left\{\left(\mathcal{I}_{2, n}\right)^{2}\right\}= \\
& \sum_{\substack{j \in J_{a} \\
j=0, j \neq n}}^{N-1}\left\{\left|I_{j-n}\right|^{2} E\left\{\left|H_{j}\right|^{2}\right\}+\frac{\left|I_{j-n}\right|^{2} E\left\{\left|G_{j}\right|^{2}\right\}}{K^{2}}\right\}+ \\
& \sum_{\substack{j \in J_{b} \\
j=0, j \neq n}}^{N-1}\left\{\frac{\left|I_{j-n}\right|^{2} E\left\{\left|H_{j}\right|^{2}\right\}}{K^{2}}+\left|I_{j-n}\right|^{2} E\left\{\left|G_{j}\right|^{2}\right\}\right\} \\
& =\sum_{j=0, j \neq n}^{N-1}\left\{\left(1+\frac{1}{K^{2}}\right)\left|I_{j-n}\right|^{2} E\left|H_{j}\right|^{2}\right\} .
\end{aligned}
$$

In (20), we have used $E\left\{\left|H_{j}\right|^{2}\right\}=E\left\{\left|G_{j}\right|^{2}\right\}$. From (20), it can be seen that the exact elements of $J_{a}$ and $J_{b}$ do not affect the interference as long as $\left|J_{a}\right|=\left|J_{b}\right|=\frac{N}{2}$.

\section{OPTIMIZATION OF $K$ OVER CODING GAIN AND PAR}

The opportunistic STBC-OFDM scheme proposed in this paper depends on the power scaling factor $K$. In this section, an optimum $K$ is derived according to the design criterion of maximizing the ratio between available coding gain on each sub-carrier and the maximum PAR. Note that $G$ and $G^{\prime}$ have the same coding gain. Therefore, we do not need to distinguish different sub-carriers. Let $\mathrm{CG}_{\mathrm{A}}$ denote the coding gain of layer $\mathrm{A}$, defined as

$$
\mathrm{CG}_{\mathrm{A}}=\min _{a 1 \neq a 2} \operatorname{det}\left(G G^{*}\right)
$$

and $\mathrm{CG}_{\mathrm{B}}$ denote the coding gain of layer $\mathrm{B}$, which is defined as

$$
\mathrm{CG}_{\mathrm{B}}=\min _{b 1 \neq b 2} \operatorname{det}\left(G G^{*}\right) .
$$

Therefore, our design criterion becomes

$$
K_{\mathrm{opt}}=\underset{K>1}{\operatorname{argmax}}\left\{\frac{\sqrt{\mathrm{CG}_{\mathrm{A}} \mathrm{CG}_{\mathrm{B}}}}{\frac{\mathrm{PAR}_{\max }}{N}}\right\},
$$

where $\frac{\mathrm{PAR}_{\max }}{N}$ is the normalized maximum PAR in (12). Fig. 2 shows the design metric as a function of $K$ for QPSK modulation. We observe that the optimum $K$ is equal to 2 .

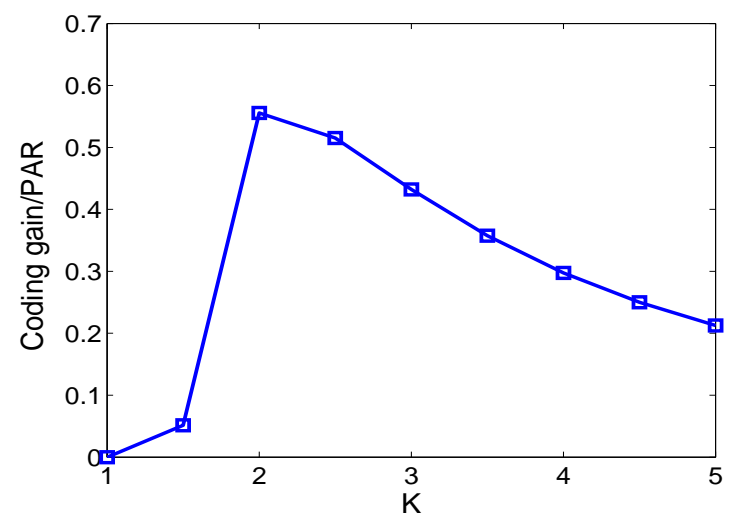

Fig. 2. Joint optimization of coding gain and PAR

\section{SIMULATION RESULTS}

In this section, we compare the performance of the following three transmission schemes:

- DEC-OFDM: $J_{a}=\emptyset$.

- Block S-DEC-OFDM: $J_{a}=\left\{0,1, \ldots, \frac{N}{2}-1\right\}$ and $J_{b}=$ $\left\{\frac{N}{2}, \ldots, N-1\right\}$.

- Interleaved S-DEC-OFDM: $J_{a}=\{1,3, \ldots, N-1\}$ and $J_{b}=\{0,2, \ldots, N-2\}$. 


\subsection{CCDF comparison}

In this simulation, $N=64$ and the OFDM symbol is oversampled by a factor of 8 . The complementary cumulative distribution function (CCDF) of the PAR is calculated over 1000,000 STBC-OFDM block realizations. Fig. 3 compares $\mathrm{CCDF}$ of the three schemes at $K=2$, where it can be

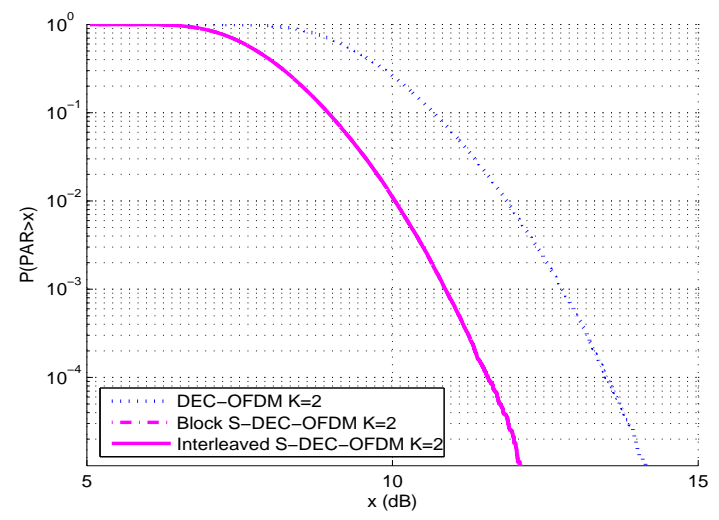

Fig. 3. PAR comparison

seen that the PAR of block S-DEC-OFDM and interleaved S-DEC-OFDM are identical and both are about $2 \mathrm{~dB}$ lower than that of DEC-OFDM.

\subsection{BER performance with ICI reduction}

In this subsection, the channels are modeled as independent 3-tap Rayleigh fading channels with an exponential power delay profile. In the simulation, we use $K=2$ and a frequency offset (normalized by the subcarrier spacing) $v=0.01$. At the receiver, the MLIC algorithm proposed in [3] is used to decode the information on each sub-carrier. When channel information is not available at the receiver, the differential detector in [8] can be used. It can be seen

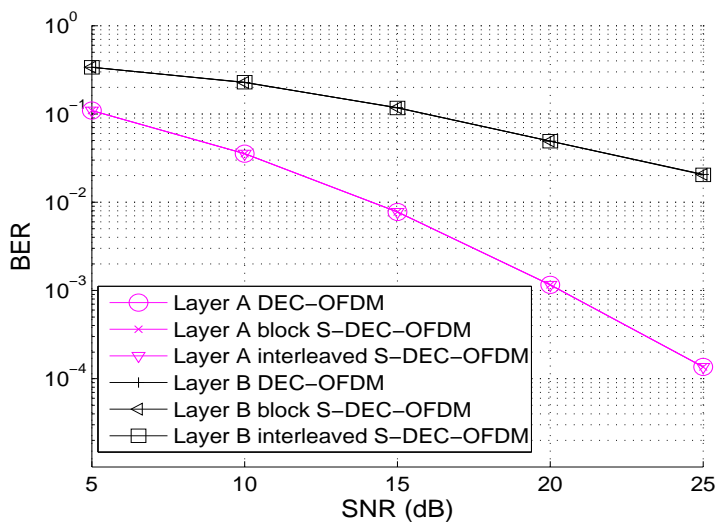

Fig. 4. Performance comparison at $K=2$ from Fig. 4 that DEC-OFDM, block S-DEC-OFDM and interleaved S-DEC-OFDM have the same BER performance, which corroborates our theoretical derivation in Section 3. However, if we include the effects of amplifier backoff and nonlinearity, the proposed S-DEC-OFDM achieves better BER performance due to its lower PAR as shown in Fig. 3.

\section{CONCLUSIONS}

In this paper, we propose an opportunistic STBC-OFDM with reduced PAR in the presence of carrier frequency offset. Our theoretical analysis shows that the lowest PAR is achieved when DEC and S-DEC are each implemented on half of the sub-carriers. ICI analysis shows that, in the presence of carrier frequency offset, sub-carriers on which DEC or S-DEC is implemented do not affect the BER performance. Finally, we optimize the scheme by selecting the optimum power scaling factor $K$ to achieve the best tradeoff between coding gain and PAR. For QPSK modulation, at the optimum power scaling factor $(K=2)$, the PAR of S-DECOFDM is $2 \mathrm{~dB}$ less than that of DEC-OFDM.

\section{REFERENCES}

[1] S. Diggavi, N. Al-Dhahir, and A.R. Calderbank, "Diversity-embedded space-time codes," in Globecom conference, December 2003, pp. 1909-1914.

[2] S. Das and N. Al-Dhahir, "New diversity-embedding space-time codes constructions," in SPAWC, July 2006.

[3] S. Das, N. Al-Dhahir, S. Diggavi, and A.R. Calderbank, "Opportunistic space-time block codes," in VTC conference, Fall 2005.

[4] V. Tarokh, N.Seshadri, and A.R. Calderbank, "Spacetime codes for high dazta rate wireless communications: performance criterion and code construction," in IEEE Transcations on Information Theory, March 1998, vol. 44, pp. 744-765.

[5] V. Tarokh, H. Jafarkhani, and A.R.Calderbank, "Spacetime block codes from orthognoal designs," in IEEE Transcations on Information Theory, July 1999, pp. 1456-1467.

[6] S. Diggavi, N. Al-Dhahir, A. Stamoulis, and A.R. Calderbank, "Great expectations : The value of spatial diversity in wireless networks," in Proceedings of the IEEE (Special Issue on Gigabit Wireless), February 2004.

[7] N. Al-Dhahir, "Overview and comparison of equalization schemes for space-time-coded signals with application to edge," in IEEE Transactions on Signal Processing Special Issue, October 2002.

[8] P. Rabiei and N. Al-Dhahir, "Differential diversityembedding space-time block coding," in Asilomar Conference, November 2006. 